\title{
Allongement chirurgical de la couronne clinique
}

\section{RÉSUMÉ}

Philippe VIARGUES

Pratique privée Paris,

87, avenue Niel,

75017 Paris.

Jean MEYER

Ex MCU-PH,

Université Paris V.

L'allongement de la couronne clinique est une intervention de chirurgie parodontale qui permet de conserver des dents dont la destruction compromet la pérennité ou dont la hauteur coronaire insuffisante ne permet pas la reconstruction. L'espace biologique doit être respecté afin de rétablir des rapports parodonto-prothétiques corrects. Une technique vestibulaire de lambeau mixte, épaisseur totale dans la partie coronaire et épaisseur partielle dans la partie apicale, est décrite. De même, une technique palatine avec une gingivectomie secondaire à l'ostéo-ectomie-ostéoplastie est proposée. Chaque étape est expliquée en fonction de la localisation de la destruction. La place de l'allongement coronaire dans le plan de traitement est développée.

\footnotetext{
Cet article a déjà fait l'objet d'une publication
} dans le $n^{\circ}$ AOS 183, de septembre 1993.

- allongement coronaire

espace biologique

lambeau d'épaisseur partiel

- limite cervicale 
La rédaction des Actualités Odonto-Stomatologiques nous a demandé de republier cet article paru dans le numéro de septembre 1993. Il est logique que nous nous posions la question de l'opportunité d'une telle re-publication quand on réalise les progrès faits par la dentisterie au cours de ces 20 dernières années...

L'élongation chirurgicale de la couronne clinique est elle toujours d'actualité ?

La réponse est bien évidemment oui. Les techniques chirurgicales qui permettent de recréer un espace biologique sain, de faciliter l'accès d'une reconstitution, d'harmoniser, d'aligner ou de déplacer une ligne du sourire sont des techniques relativement simples qui font absolument partie du quotidien de tous les praticiens.

En revanche, la question est beaucoup plus mesurée quand il s'agit de récupérer une dent ou une racine compromise. Bien évidemment, à chaque fois que cela est possible, toute solution permettant la conservation d'un organe dentaire doit être privilégiée. Mais, s'il y a 20 ans et plus il fallait parfois s'autoriser certaines «acrobaties» pour conserver une dent stratégique, il est actuellement raisonnable d'envisager, aussi, une solution implantaire. La difficulté aujourd'hui n'est plus tant liée à «l'acrobatie» possible qu'à la décision à prendre face à l'alternative conserver ou extraire et poser un implant. Incontestablement, en 2009, la solution implantaire est une solution fiable et élégante et qui semble simple. Cette apparente facilité ne doit pas nous faire choisir l'implant trop rapidement. Tout le problème réside aujourd'hui dans le choix de la voie à suivre. Ce choix doit être fondé sur une connaissance et une réflexion qui donnent la priorité au patient, à son bien-être, à la fiabilité tant fonctionnelle qu'esthétique et surtout à la valeur du rapport coût/durabilité de l'option thérapeutique envisagée et tant pis si ce choix n'est pas le plus simple.

Bien que d'indications plus réduites, l'élongation chirurgicale de la couronne clinique reste d'actualité sachant, de plus, que la bonne maitrise de cette technique est sûrement le meilleur moyen de savoir quand il ne faut pas y avoir recours...

Philippe Viargues

\section{Introduction}

L'allongement chirurgical de la couronne clinique est une technique qui permet d'utiliser une ou plusieurs dents dont la hauteur coronaire est insuffisante pour une reconstruction prothétique ou dont les destructions corono-radiculaires atteignent l'espace biologique et compromettent leur restauration.

L'espace biologique, clef de voûte des rapports parodonto-prothétiques, représente la dis- tance moyenne entre le fond du sulcus et la crête osseuse. Cette entité, composée de l'épithélium de jonction et de l'attache conjonctive, a été définie par Sicher en 1959, puis étudiée et quantifiée par Gargiulo et al. en 1961. Les valeurs moyennes obtenues par ces auteurs après 325 mesures sont respectivement de: $0,97 \mathrm{~mm}$ pour l'épithélium de jonction et $1,07 \mathrm{~mm}$ pour l'attache conjonctive. Ainsi l'espace biologique présente une valeur moyenne 
de 2,04 $\mathrm{mm}$, la profondeur du sulcus étant de $0,69 \mathrm{~mm}$.

Dans le cadre parodonto-prothétique, cette évaluation signifie que la distance comprise entre la crête osseuse et la limite la plus apicale d'une reconstruction ne devrait jamais être inférieure à 2,04 mm.

Dans les cas d'allongement coronaire, les auteurs ont proposé des valeurs comprises entre $3 \mathrm{~mm}[8,12,16]$ et $5 \mathrm{~mm}$ ou plus $[22,24]$. La littérature française préconise une distance comprise entre 2,5 et $3 \mathrm{~mm}[1,3,6,7,11]$.

Étienne et Joly [4] constatent une tolérance variable et réfutent la notion impérative de $3 \mathrm{~mm}$. Viargues et Meyer [23] proposent un concept d'espace biologique et d'allongement coronaire variable en fonction du contexte et du cas.

\section{Indications et contre-indications}

\section{Indications}

Les indications sont celles de toute destruction dentaire empiétant sur le système d'attache, quelle que soit la face concernée et en fonction de l'intérêt stratégique de cette dent. Cette intervention ne peut se concevoir que conjointement à une endodontie de qualité et avec un environnement parodontal sain ou assaini. Si cet assainissement est réalisé dans le même temps que l'allongement coronaire, la phase de temporisation post-chirurgicale sera plus longue. Bien que le traitement du système canalaire précède en principe l'allongement coronaire, dans certains cas, cette intervention peut être effectuée en premier lieu pour des nécessités de rigueur endodontique.

Ces indications peuvent être modulées en fonction du rôle stratégique de la dent concernée. Le degré d'exigence quant à la hauteur de racine résiduelle n'est pas le même pour une dent appui de bridge ou pour une racine destinée à recevoir une chape sous une prothèse adjointe.

\section{Contre-indications}

Les contre-indications d'ordre général restent valables comme pour toute chirurgie buccale. Les contre-indications loco-régionales concernent le niveau d'hygiène du patient, l'intérêt particulier de la dent, ou le risque d'atteinte du support parodontal des dents voisines. L'allongement coronaire chirurgical est également contre-indiqué si une technique orthodontique, extrusion ou égression, en particulier au secteur antérieur, est techniquement réalisable. Les contre-indications peuvent être également d'ordre anatomique et concernent essentiellement deux points : la proximité des zones interradiculaires et la valeur du futur rapport racine/couronne. Classiquement, ce rapport doit être au minimum de 1/1 et, pour certains auteurs, une diminution de ce rapport à une valeur inférieure est une contre-indication absolue [3].

Ce rapport 1/1, concernant les proportions minimales couronne/racine des ancrages prothétiques, semble cependant pouvoir supporter certains aménagements [9]. Ainsi, si au 
niveau de la crête osseuse normalement située $2 \mathrm{~mm}$ apicalement à la ligne de jonction émail/cément s'ajoute la valeur de l'allongement coronaire de $3 \mathrm{~mm}$ afin de restaurer l'espace biologique, le rapport couronne/racine $s^{\prime}$ inverse et devient inférieur à 1 .

En effet, après allongement, le nouveau niveau osseux se situe au minimum à $5 \mathrm{~mm}$ de la jonction émail/cément. Ainsi, compte tenu des longueurs radiculaires situées entre 13 et $17 \mathrm{~mm}$, pour des valeurs totales de 23 à
$27 \mathrm{~mm}$ [21] aucune dent, après chirurgie, ne présente un rapport racine/couronne égal ou supérieur à 1.

Comme pour toute chirurgie buccale, les plus grandes précautions seront prises quand l'intervention se déroulera dans une zone proche d'éléments anatomiques à épargner ou à protéger particulièrement, comme, par exemple, l'émergence du nerf dentaire inférieur au trou mentonnier.

\section{Techniques chirurgicales}

Les techniques sont fonction de la localisation de la destruction.

\section{Destruction intéressant la face vestibulaire}

(fig. 1)

Cette destruction peut être consécutive à une fracture (fig. 2) ou bien d'origine carieuse (fig. 3). Après anesthésie locale, deux incisions parallèles, verticales sont réalisées de part et d'autre de la dent concernée. Un lambeau d'épaisseur totale est récliné dans la partie coronaire puis prolongé par une dissection en épaisseur partielle dans la partie apicale (fig. 4). Ce lambeau mixte présente plusieurs intérêts :

- pour Staffileno [18], une meilleure adaptation du lambeau serait obtenue, accélérant ainsi la formation du réseau de fibrine, élément précurseur nécessaire aux connexions conjonctives [15] ;

- la conservation du périoste à la surface osseuse constitue une protection vis-à-vis de la résorption $[11,14,18]$;
- la conservation des fibres saines à la surface de la racine et des terminaisons conjonctives au niveau de la surface osseuse favorise un pontage fibreux [19] ;

- la conservation du périoste permet de réaliser des sutures périostées extrêmement favorables à l'immobilisation du lambeau et à sa cicatrisation ;

- de plus, le positionnement apical d'un lambeau d'épaisseur totale sur une zone toujours périostée conduit à une augmentation en épaisseur du conjonctif, élément favorable avant la pose d'une prothèse fixée.

L'ostéo-ectomie-ostéoplastie est ensuite réalisée et doit permettre (fig. 5) :

- de fixer une limite de préparation définitive ;

- de ménager, entre cette limite et l'os, une zone pouvant varier de 1 à $3 \mathrm{~mm}$ [23], correspondant à l'espace biologique ;

- d'harmoniser les contours osseux des dents collatérales. 


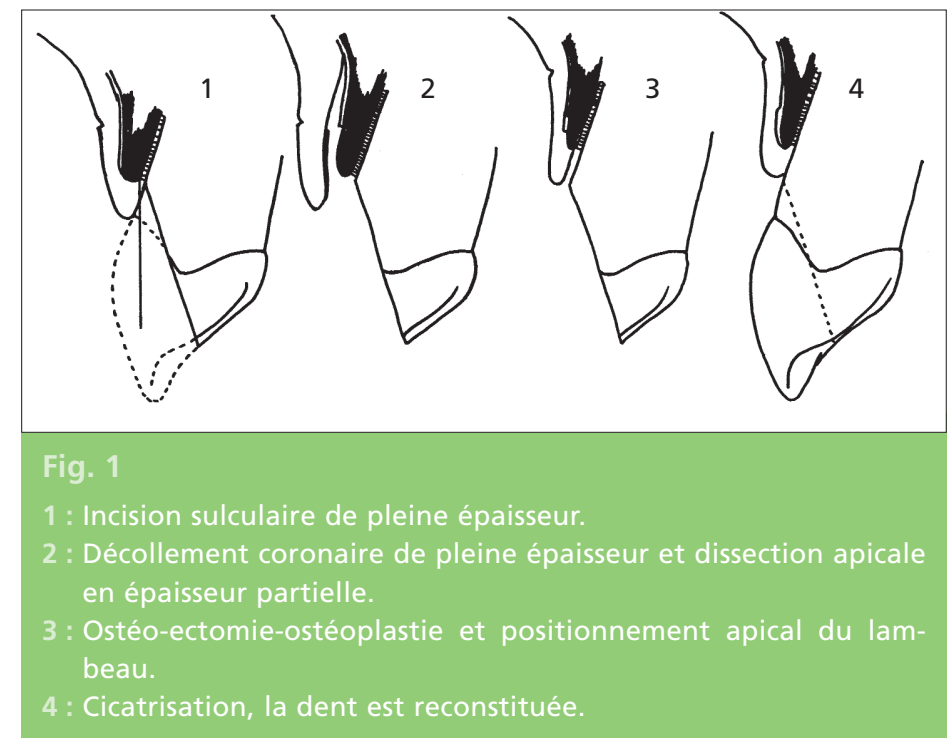

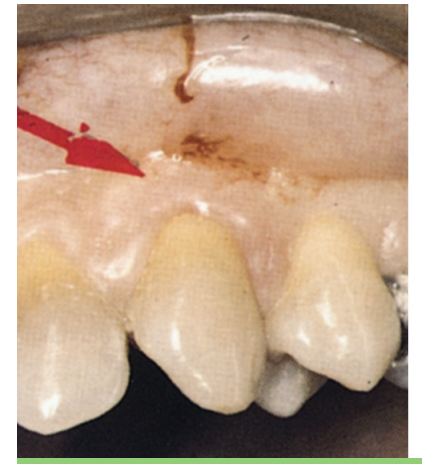

Fig. 2

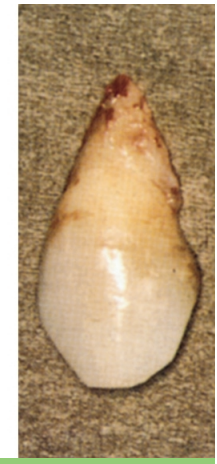

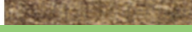
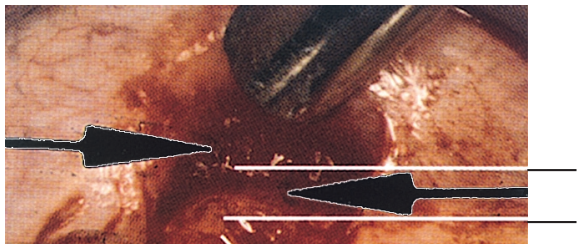

épaisseur partielle épaisseur totale

trait de fracture sous le niveau osseux

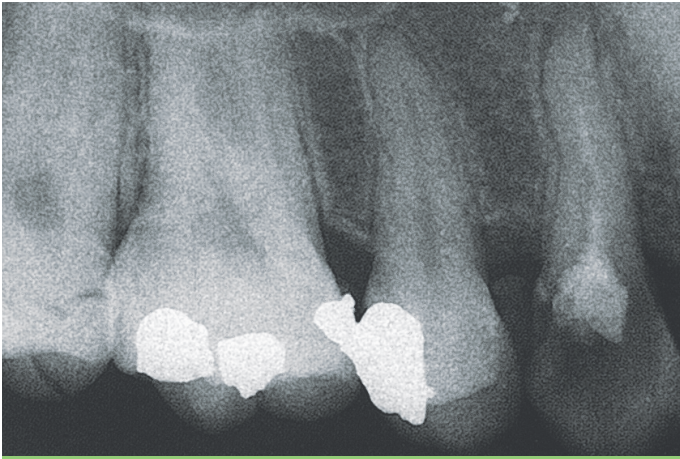

Fig. 3

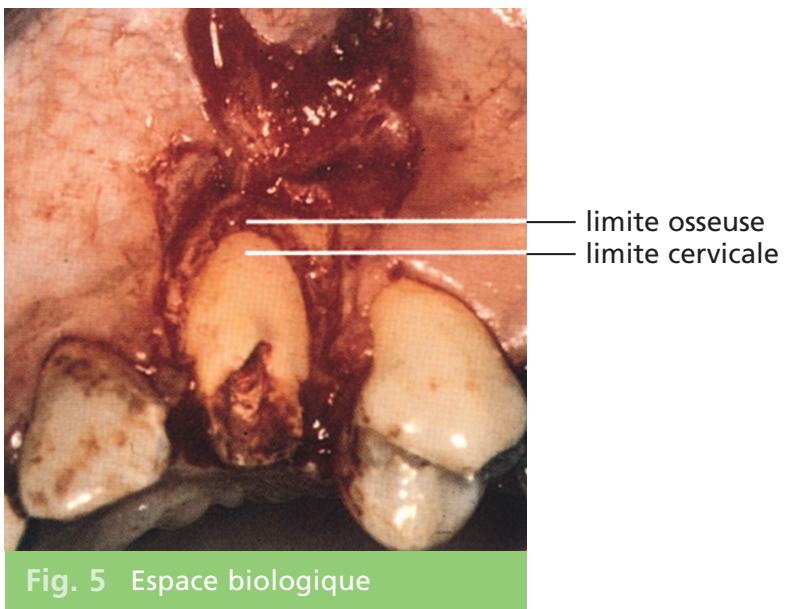




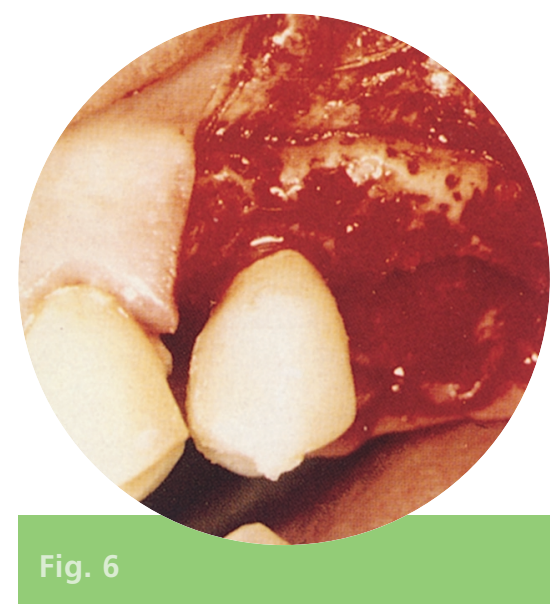

Une couronne provisoire doit être réalisée ou rebasée en peropératoire avec une adaptation méticuleuse aux limites de préparation de la racine résiduelle pour optimiser la qualité de la cicatrisation (fig. 6). Cette couronne sera scellée provisoirement en fin d'intervention.

Le lambeau est suturé au périoste, apicalement par rapport à sa position d'origine, à I'aide de points latéraux et/ou apicaux (fig. 7). Des sutures matelassées peuvent également être envisagées afin d'obtenir l'immobilisation et le placage le plus parfait possible du lambeau.

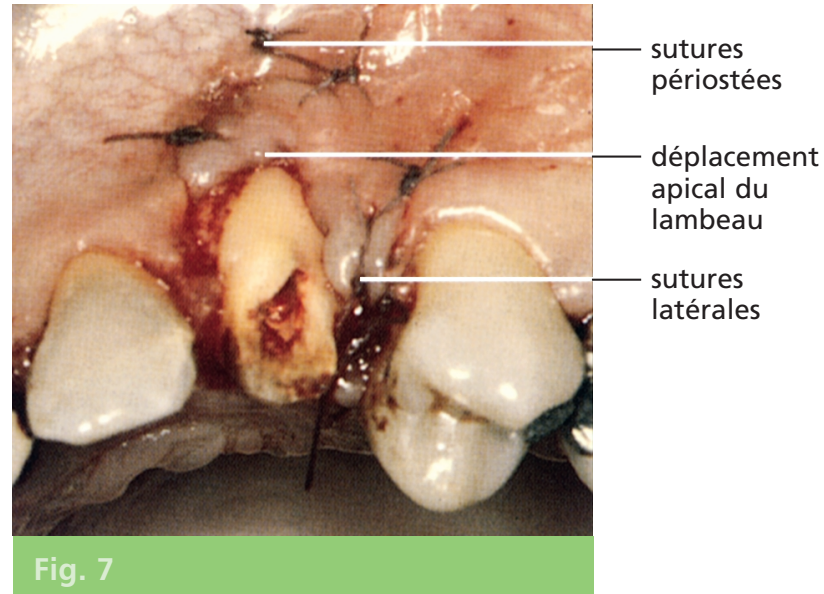

Destruction intéressant

la face palatine seule

(fig. 8 et 9)

Dans un premier temps, une incision palatine, sulculaire, en pleine épaisseur est pratiquée et se prolonge sur au moins une dent de part et d'autre de la dent concernée (fig. 10). Après décollement de ce lambeau palatin, I'ostéoectomie-ostéoplastie est réalisée. Elle doit viser les mêmes objectifs que dans l'abord vestibulaire. À ce stade, il faut s'assurer que l'os se termine en pente douce au contact de la racine.

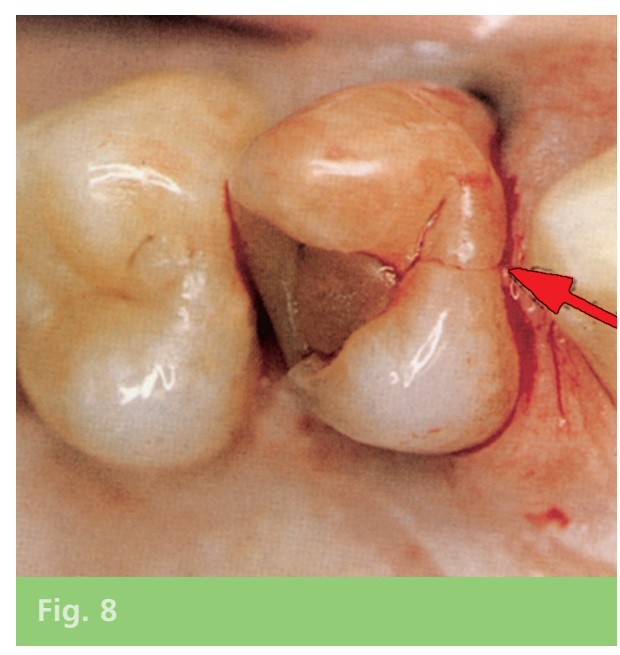




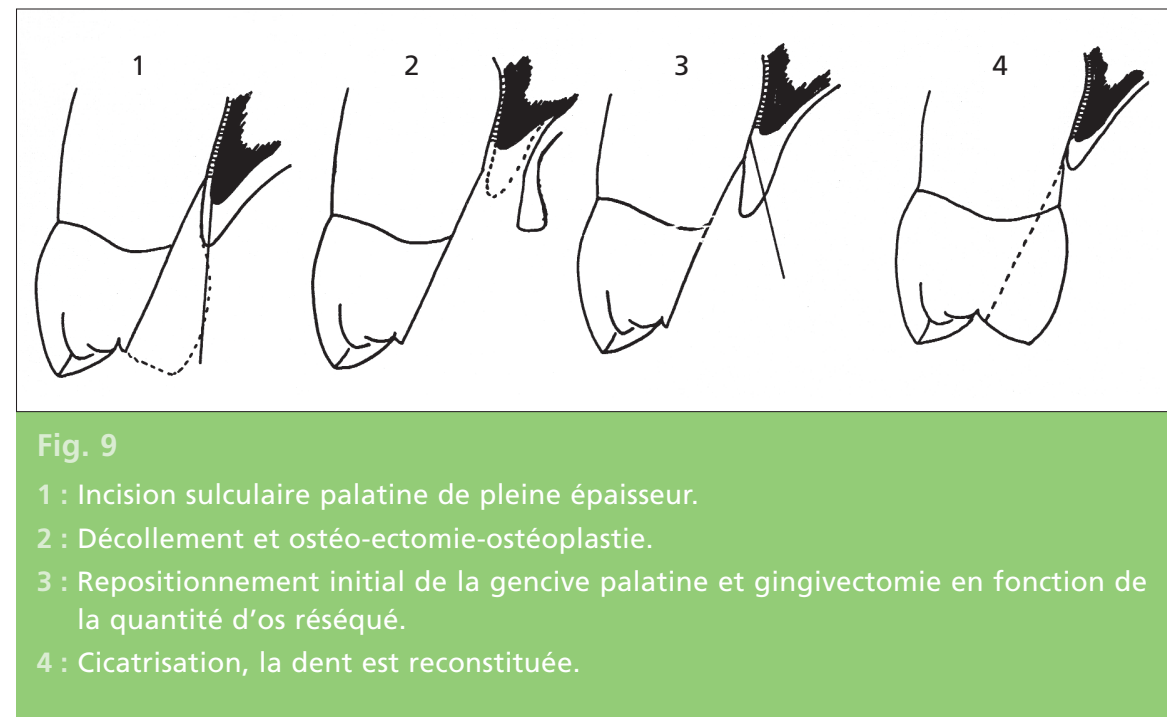

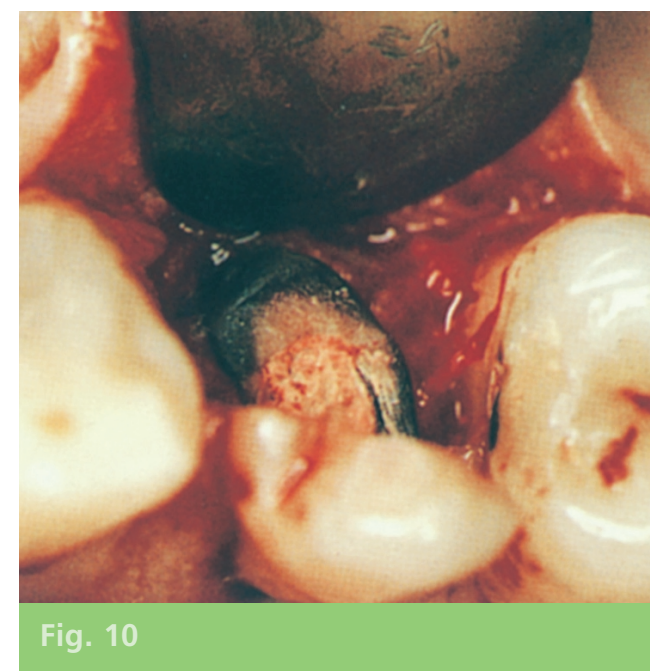

Le déplacement d'un lambeau palatin étant anatomiquement impossible, son adaptation à la racine ne peut se faire qu'au moyen d'une gingivectomie. En effet, après avoir réappliqué le lambeau, il est possible de mesurer, à l'aide d'une sonde graduée, la quantité de gencive comprise entre son bord marginal et la limite de préparation fixée pour la future reconstruction. Le niveau où doit se situer cette gingivectomie est ainsi

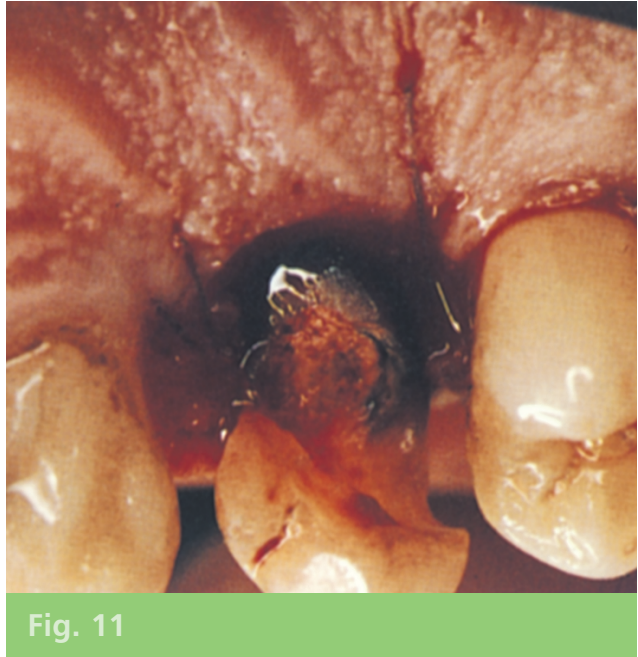

objectivé. Celle-ci éliminera la partie de gencive en excès. Parallèlement à l'os qui doit se présenter en pente douce au contact de la racine, la gencive doit également être affinée au détriment de la face conjonctive afin de recréer des rapports "racine/os/gencive» harmonieux.

Les sutures, de type matelassier, plaqueront parfaitement le lambeau palatin sur son site (fig. 11). 


\section{Destruction périphérique}

(fig. 12)

Lors de la destruction totale de la couronne, l'abord chirurgical doit combiner les deux techniques décrites précédemment (fig. 13). Les zones des papilles seront éliminées afin de pouvoir aborder la crête osseuse interdentaire et réaliser les retouches osseuses avec les mêmes objectifs que précédemment. Cette zone cicatrisera par seconde intention et épithélialisation secondaire.

Ce type d'intervention se termine par la pose d'un pansement parodontal. Les suites opératoires sont généralement sans complications. L'antibiothérapie n'est pas nécessaire en dehors des indications générales. Les sutures seront déposées après une semaine. Les résultats, en présence de reconstitutions non iatrogènes, sont stables dans le temps (fig. 14 à 16).

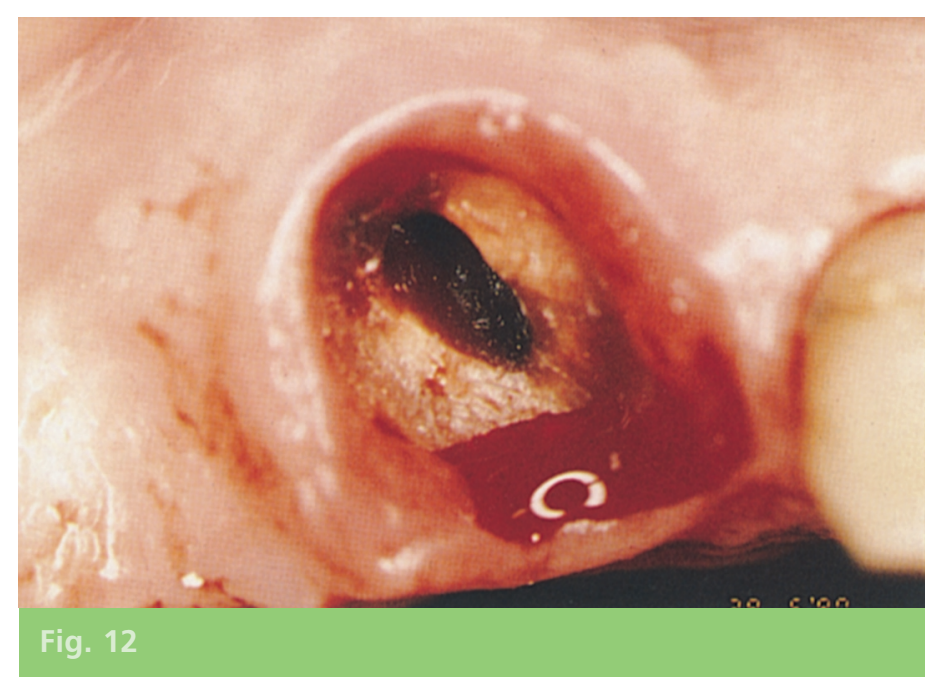

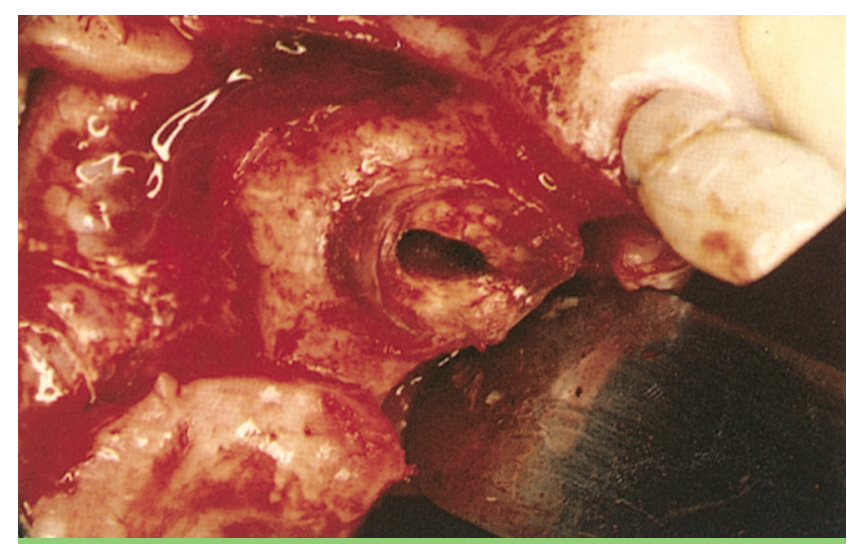

Fig. 13

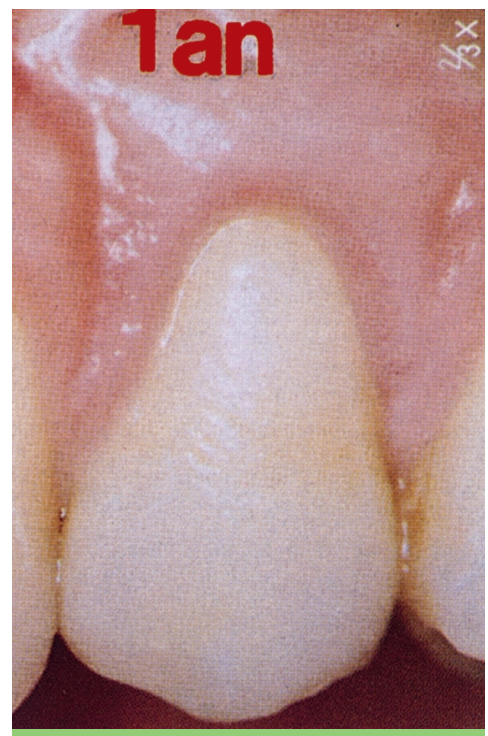

Fig. 14 Résultats à 1 an du cas des figures 2, 4, 5, 7 . 

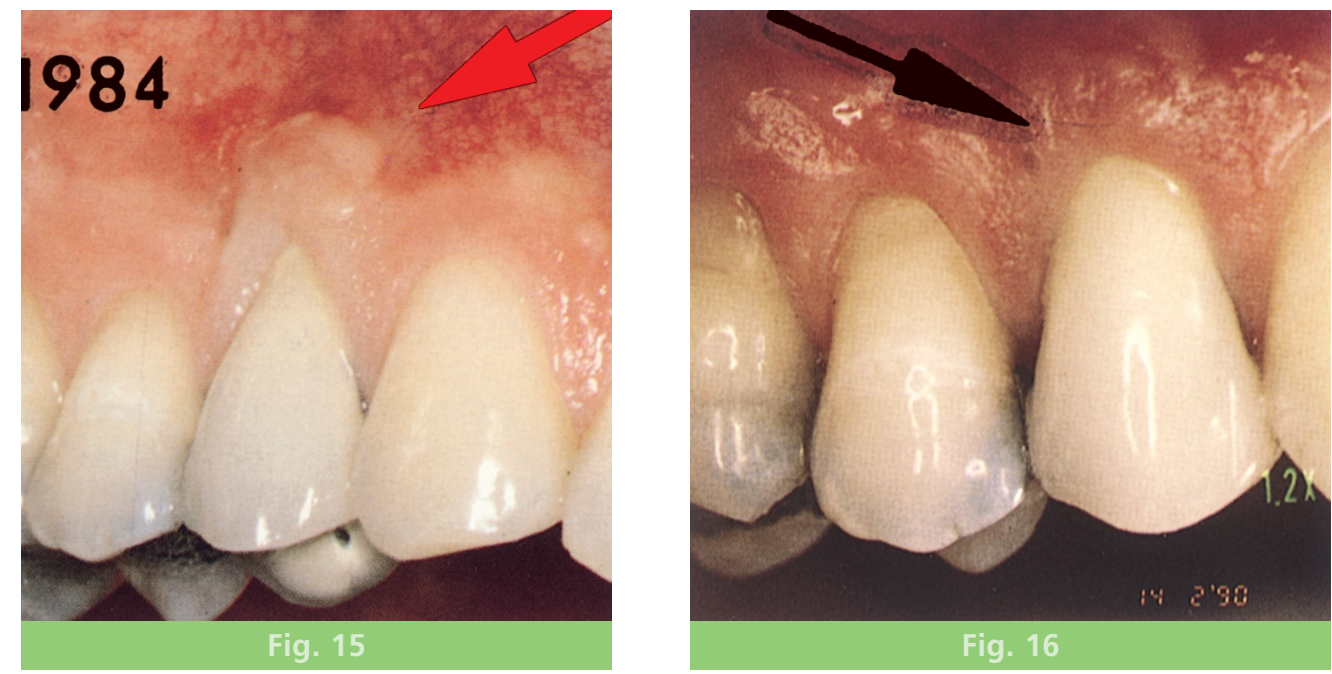

Fig. 15 et 16 Résultats à 18 mois et 7 ans d'un cas similaire.

\section{Place de l'allongement coronaire dans le plan de traitement}

Cette place dépend du type de cas à traiter.

> Cas unitaire, en l'absence de maladie parodontale

Soit l'endodontie est correcte ou peut être réalisée d'emblée et elle sera alors un préalable à la décision de conservation de la dent considérée; soit le déficit en structure dentaire est tel que l'endodontie ne peut pas être réalisée dans de bonnes conditions et l'allongement coronaire devient le préalable à l'endodontie. Récupération parodontale et endodontique d'une racine sont indissociables. Une des deux techniques se plaçant, selon le cas, au service de l'autre. Ces deux conditions de conservation sont impératives.

\section{> Cas unitaire ou plural avec présence de maladies parodontales}

L'allongement coronaire ne peut prendre place qu'après un enseignement réussi $d u$ contrôle de plaque par le patient et la phase de traitement étiologique des maladies parodontales. Dans ce cas de figure, le plan de traitement ne peut se décider qu'en fonction de l'endodontie, de l'environnement parodontal et de la réponse aux premiers traitements parodontaux.

L'allongement réalisé, une couronne provisoire parfaitement ajustée aux limites définitives de la future restauration doit être laissée en place pour une durée de 6 mois. En effet, les travaux de Parma-Benfenati et al. [13] montrent, après violation de l'espace biologique, l'apparition d'un réseau de fibres dento-périostées qui vont s'opposer à la progression de l'inflammation avant le $3^{e}$ mois. Tal et al. [20] montrent que s'il persiste une lyse osseuse entre le $3^{e}$ et le $6^{e}$ mois, celle-ci n'est pas statistiquement significative. Ainsi le processus de lyse diminuerait dans le temps 
pour s'arrêter vers le $6^{\mathrm{e}}$ mois. Enfin Bragger et al. [2] montrent qu'après un allongement de $3 \mathrm{~mm}, 12 \%$ des sites peuvent présenter une récession gingivale comprise entre 2 et $4 \mathrm{~mm}$

\section{Conclusion}

L'allongement coronaire est une technique extrêmement fiable. Si les indications sont bien posées et la réalisation de l'intervention correcte, le succès paraît assuré. Le respect de I'espace biologique, d'un temps de cicatrisation suffisant, et la réalisation d'une prothèse de qualité sont les éléments indispensables à la réussite d'un tel traitement. Ce type d'inter- entre la $6^{\mathrm{e}}$ semaine et le $6^{\mathrm{e}}$ mois. Ces trois observations justifient l'attente de 6 mois nécessaire avant la réalisation de la prothèse définitive. vention permet d'envisager la conservation de dents très compromises. Mais le résultat est en relation avec le respect indispensable des indications et des contre-indications. L'allongement de la couronne clinique est une technique chirurgicale qui a ses propres limites et qui ne doit être utilisée que dans le cadre d'un plan de traitement parfaitement défini.

\section{Bibliographie}

1. Alcouffe F.

L'élongation coronaire.

Inform Dent

1992;32:2747-2750.

2. Bragger $U$, Lauchenauer $D$, Lang NP.

Surgical lengthening

of the clinical crown.

J Clin Period 1992;19:58-63.

3. Caire T, Saffar JL. Chirurgie parodontale préprothétique :

l'allongement coronaire.

J Parod 1984:3:439-450.

4. Étienne D, Joly R.

Chirurgie préprothétique

et traitement

de l'édentement.

J Parod 1992;11:167-177.

5. Gargiolo AW, Wentz F, Orban B.
Dimensions and relations of dentogingival junction in humans.

J Period 1961;42:8-12.

6. Genon P.

Chirurgie parodontale en préparation à la prothèse scellée. Actualités Odonto-Stomat 1986;153:39-62.

7. Giovannoli JL, NGuyen MA Aménagement de l'espace biologique par extrusion orthodontique. Réalités cliniques 1992;3(2):173-183.

8. Ingberg FJS, Rose LF, Coslet JG.

The biologic width A concept in periodontics and restorative

dentistry.

Alpha-Omega

1977; 10:62-65.

9. Lindhe J.

Manuel

de parodontologie

clinique.

Paris, Éditions CdP, 1986.

10. Meyer J.

Allongement de la couronne clinique. Rev Odonto-Stomat 1984;105-108.

11. Meyer J, Huynh C. Rétention des fibres et lambeau d'épaisseur partielle dans le traitement des lésions osseuses. J Parod 1987;6:225-235. 
12. Nevins M, Skurow HM.

Emplacement intracréviculaire du bord prothétique, largeur biologique et maintenance du bord gingival. Rev Int Parod \& Dent Rest 1984;3:31-49.

13. Parma-Benfenati $S$, Fugazzotto P, Ruben M. Incidence de la situation des bords d'une restauration prothétique sur le devenir post-chirurgical et la nature du parodonte, 1re partie.

Rev Int Parod \& Dent Rest 1985;6:31-50.

14. Pfeiffer JS.

The reaction of alveolar bone to flap procedure in man. Periodontics 1965;3:135-140.

15. Polson AM, Proye MP. Fibrin linkage: a precursor for new attachment.

J Period 1983;54:141-147.

16. Ross SE, Gargiulo A, Crosseti HW, Phillip D. Traitement chirurgical de l'interface alvéolo-prothétique, $2^{\mathrm{e}}$ partie.

Rev Int Parod \& Dent Rest 1983;4:8-19.

17. Sicher $\mathrm{H}$.

Changing concepts

of the supporting

dental structure.

Oral Surg Oral Med

Oral Pathol 1959;12:31-35.

18. Staffileno $\mathrm{H}$.

Significant differences and advantages following the full thickness and split thickness flaps. J Period 1974;45:421-425.

19. Stahl SS.

Speculation on periodontal attachment loss.

J Clin Period 1986;13:1-5.

20. Tal $H$, Soldinger $M$, Dreiangel A, Pitaru S.
Réponse à l'agression

parodontale chez le chien :

suppression

de l'attache gingivale

et mise en place

supra-crestale

d'obturations en amalgame.

Rev Int Parod \& Dent Rest

1988;3:45-54.

21. Tallec $P$.

E.M.C.

12:1969 - 22003 C 10, C 20,

D 10, D 20.

22. Van Der Velden U.

Regeneration

of the interdental

soft tissues following

denudation procedures.

J Clin Period 1982;9:455-459.

23. Viargues $P$, Meyer J.

À paraître.

24. Wagenberg B, Eskow R, Langer B.

Exposing adequate

tooth structure

for restorative dentistry.

Rev Int Parod \& Dent Rest

1989;9(5):322-331.

\section{SUMMARY}

\section{Surgical elongation of the clinical crown}

Philippe VIARGUES,

Jean MEYER

\section{Keywords \\ - crown lengthening \\ - biological width \\ - partial thickness flap \\ - cervical limit}

Elongation of the clinical crown is a periodontal surgical technique enabling to preserve teeth whith advance destruction or insufficient crown height. The biological space should be respected in order to reestablish the correct perio-prosthetic relationships. A buccal technique employing a mixed flap of full thickness in the crown section and partial thickness in the apical section is described. Likewise, a palatine technique with gingivectomy secondary to osteoectomy-osteoplasty is proposed. Each step is explained according to the site of the damage. The place of crown elongation in the treatment plan is discussed. 


\section{Association d'Enseignement d'Odontologie et de Stomatologie}

\section{LA VIE DE L'ASSOCIATION}

PRÉSIDENTS D'HONNEUR :

Pierre CERNÉA ${ }^{\dagger}$, Jean OUVRARD ${ }^{\dagger}$, Michel BENOIST ${ }^{\dagger}$

\section{MEMBRES D'HONNEUR :}

A. RICHARD ${ }^{\dagger}$, H. LENTULO ${ }^{\dagger}$,

A. LAMBERT, P. GONON ${ }^{\dagger}$,

J.-P. RAGOT ${ }^{\dagger}$

Ch. DESCROZAILLES,

G. FICHELLE ${ }^{\dagger}$

\section{CONSEIL D'ADMINISTRATION}

PRÉSIDENT : Hubert OUVRARD

VICE-PRÉSIDENTS :

LUC CHIKHANI,

Jean-François LEGRAND

SECRÉTAIRE GÉNÉRAL :

Frédéric LARCHÉ

TRÉSORIER :

Gérard PASQUET

MEMBRES :

Jacques-Charles BERTRAND,

Danielle BUCH, Jean BUQUET,

Estelle FAVRE, Jean MEYER,

Guy PRINC, Valérie TRAVERT

\section{COMITÉ SCIENTIFIQUE}

PRÉSIDENT : LUC CHIKHANI

VICE-PRÉSIDENT : Hubert OUVRARD

SECRÉTAIRE : Frédéric LARCHÉ

MEMBRES : Christophe BONNEFOY, Estelle FAVRE, Gilles FLEURIDAS, Thierry GUÉRIN, Jean-François LEGRAND, Frank LEVAVASSEUR, Hervé MISSISTRANO, Gérard PASQUET, Thierry PIRAL, Valérie TRAVERT

ANCIENS PRÉSIDENTS : P. CERNÉA ${ }^{\dagger}$, P. HENNION, H. LENTULO ${ }^{\dagger}$, M. LEPOIVRE ${ }^{\dagger}$ A. MARMASSE ${ }^{\dagger}$, A. LAMBERT, R.-R. RIGOLET ${ }^{\dagger}$, R. BATAILLE', L.-A. STIEGLER ${ }^{\dagger}$, F. BOUCHON ${ }^{\dagger}$, Ch. DESCROZAILLES, P. FRIEZ ${ }^{\dagger}$, F. BROCHERE ${ }^{\dagger}$, A. RICHARD ${ }^{\dagger}$, R.-L. NINET', M. CHATEAU, P. COUSTAING, A. MUGNIER, R. WEILL', C. CREPY, J. OUVRARD ${ }^{\dagger}$, Y. COMMISSIONAT, B. DANGY ${ }^{\dagger}$, F. GARLOPEAU ${ }^{\dagger}$, J.-L. DEPHILIPPE, J. VIGNEUL, H. PETIT, M. BENOIST ${ }^{\dagger}$, G. FICHELLE, J. LAUFER, J.-P. SANTORO, P. LAUDENBACH ${ }^{\dagger}$, P. DARGENT ${ }^{\dagger}$, CI. SCHUHMANN, F. MAESTRONI ${ }^{+}$, J.-P. DEFFEZ, H. OUVRARD, D. RIGOLET, J. BUQUET, J.$\begin{array}{lll}\text { P. RAGOT } & \text { R. BUGUGNANI, P. BORDAIS, }\end{array}$ J.-M. LAURICHESSE', J.-Ch. BERTRAND, J.-F. LEGRAND, G. PASQUET, J. DICHAMP， D. BUCH， A. DEBOISE', C. BOZON, F. LARCHÉ, M. AMORIC, G. PRINC, V. TRAVERT, J.-P. LÉZY, F. LEVAVASSEUR, E. FAVRE, H. MISSISTRANO, T. PIRAL, C. BONNEFOY

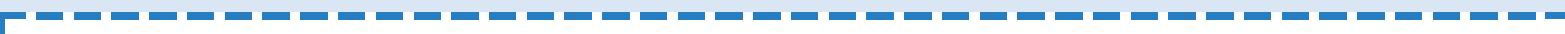

\section{BULLETIN D'ADHÉSION À L'A.E.O.S.}

Oui, je souhaite devenir membre de I'A.E.O.S. Ci-joint le règlement de ma cotisation annuelle 2009 : $20 €$ au lieu de $60 €$ (offre réservée aux nouveaux abonnés des A.O.S.).

Je règle par chèque à l'ordre de l'A.E.O.S.

$\mathrm{Dr}:$

Prénom :

Adresse

Code postal :

Ville :

Téléphone :

Fax :

Bulletin à retourner à : I'Association d'Enseignement d'Odontologie et de Stomatologie (A.E.O.S.) 179, rue Saint-Honoré - 75001 Paris - Tél. 0142605032 - Fax 0147034643 